SCIENTIFIC LETTER

\title{
Augmented metabolic control improves myocardial diastolic function and perfusion in patients with non-insulin dependent diabetes
}

\author{
H von Bibra, A Hansen, V Dounis, T Bystedt, K Malmberg, L Rydén
}

Heart 2004;90:1483-1484. doi: 10.1136/hrt.2003.020842

D iastolic dysfunction and compromised myocardial blood flow are characteristic findings in patients with non-insulin dependent diabetes. We conducted a pilot trial to test the hypothesis that intensified metabolic control would improve myocardial function and perfusion in patients with non-insulin dependent diabetes.

\section{DESIGN AND METHODS}

Twenty five patients with non-insulin dependent diabetes (19 men, mean age 60 (9) years, diabetes duration 8 (7) years) were subjected to intensified metabolic control based on an increased dose of insulin (group A: $\mathrm{n}=16$, fasting $\beta$ glucose $11(4) \mathrm{mmol} / \mathrm{l}$ ) or of oral treatment (group B: $\mathrm{n}=9$, fasting $\beta$ glucose 11 (3) $\mathrm{mmol} / \mathrm{l}$ ). Exclusion criteria were a moderate to severe degree of diabetic microvascular complications, clinical signs of heart failure, valvar disease, severe hypertension, and concomitant hepatic or renal dysfunction. A further eight patients were studied as controls during unchanged medication (mean age 62 (6) years, fasting $\beta$ glucose 8 (2) $\mathrm{mmol} / \mathrm{l}$ ).

Evidence of coronary artery disease was assessed based on coronary angiography or stress echocardiograms (dobutamine $10-40 \mathrm{mg} / \mathrm{kg} / \mathrm{min}$ ). All patients were trained to measure blood glucose with a reflectometer (Accutrend Sensor, Roche Diagnostics, Stockholm, Sweden). Self reported concentrations of fasting blood glucose averaged from three days before each visit served as a reference for glucose control. Myocardial perfusion and function were assessed by ultrasound technology at rest and during maximum vasodilatation (dipyridamole $0.84 \mathrm{mg} / \mathrm{kg}$ ) before (visit l) and three weeks after (visit 2) modification of antidiabetic treatment.

Pulsed Doppler myocardial velocity tracings were obtained (System FiVe VingMed-General Electrics, Milwaukee, Wisconsin, USA) and analysed as previously described in detail '. Systolic, early diastolic, and late diastolic peak velocities were determined and averaged from three consecutive cardiac cycles.

Myocardial contrast echocardiography (HDI 5000, ATL Inc, Bothell, Washington, USA) was performed with a broadband transducer at $1.77 \mathrm{MHz}$ and pulse inversion grey scale imaging at a mechanical index of 1.2 and analysed as previously described. ${ }^{12}$ The contrast agent Levovist (Schering, Berlin, Germany) was infused intravenously ( $600 \mathrm{mg} / \mathrm{min}$ for seven minutes). Five end systolic images were recorded at each of the incremental microbubble replenishment intervals of one, two, three, four, six, and eight cardiac cycles. The relation between background subtracted myocardial signal intensity and the increasing replenishment intervals was used to calculate the two primary components of myocardial flow, with the initial slope providing a measure of flow velocity and plateau signal intensity that correlates with myocardial capillary blood volume. $^{3}$ The averaged signal intensities at the replenishment times six and eight cardiac cycles were normalised for the signal intensity of the left ventricular cavity (percentage) and taken as an index of myocardial capillary blood volume.

All analyses were performed by two observers blinded to treatment group allocation.

The ethics committee of the Karolinska Hospital, Stockholm, approved the study protocol. All participants gave their written informed consent.

\section{RESULTS}

Baseline characteristics as regards diabetes and its complications were comparable between the two groups and so were additional risk factors for cardiovascular disease such as hypertension (63\%), hyperlipidaemia (54\%), and smoking $(21 \%)$. At visit 1 , the antidiabetic treatment was similarly
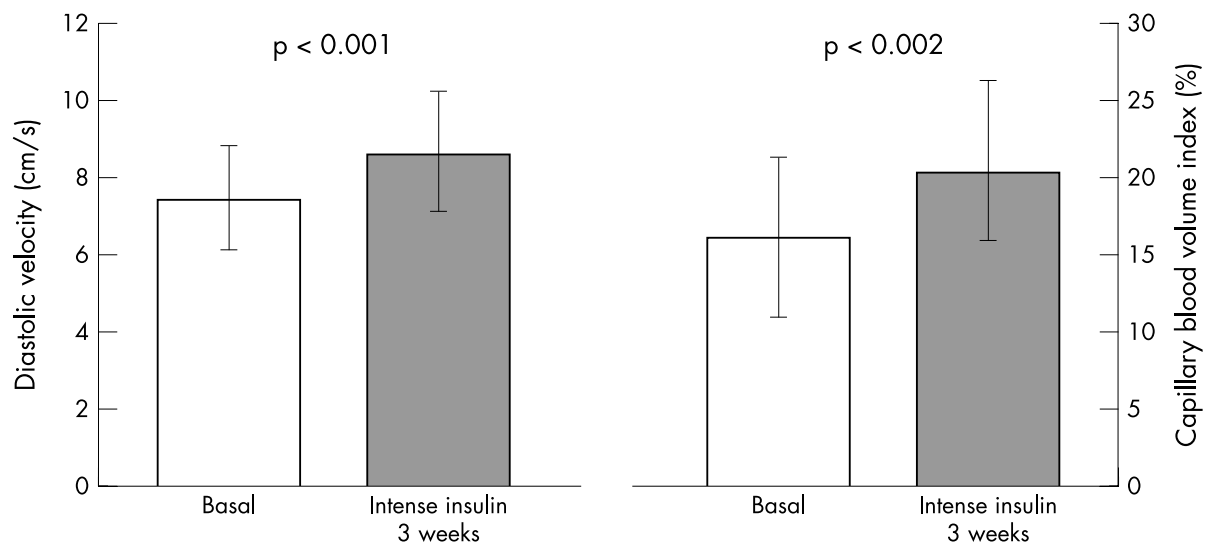

Figure 1 Diastolic velocity (left) and coronary blood volume index (right) in patients in group A, who were treated with intensified insulin, from visit 1 (basal) to visit 2 . There was a significant improvement in both diastolic velocity $(p<0.001)$ and coronary blood volume index $(p<0.002)$ with improved glycaemic control. 

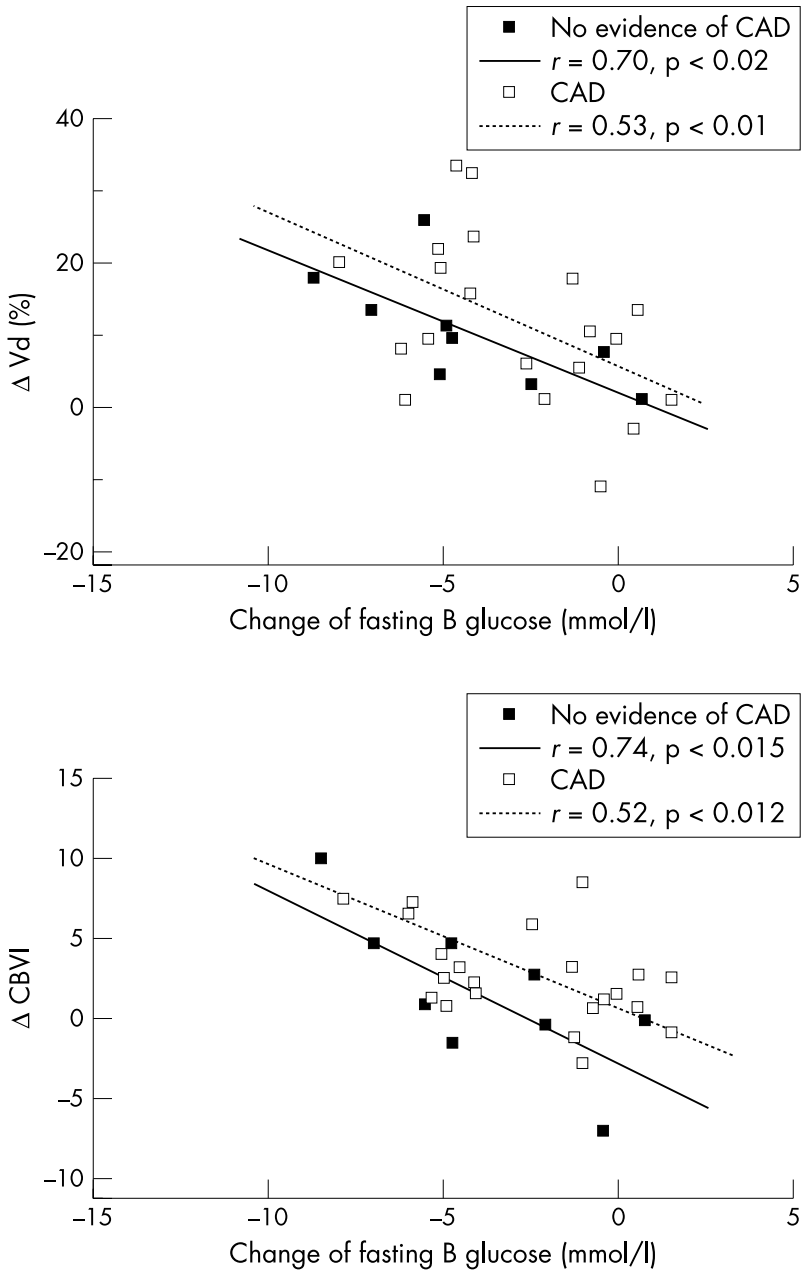

Figure 2 There was a significant correlation between change of fasting blood glucose and change of diastolic myocardial velocity ( $\Delta \mathrm{Vd}$; top) and change of myocardial capillary blood volume index ( $\triangle \mathrm{CBVI}$;

bottom) in the total patient cohort at visit 2 . These correlations remained significant in the subgroups of patients without or with evidence of coronary artery disease (CAD).

distributed (group A: oral 75\%, insulin 50\% versus group B: oral $89 \%$, insulin $67 \%$ ). At visit 2 , all patients in group A were given insulin by definition (oral 69\%; insulin 100\% with an increase of the average insulin dose from 24 (31) to 34 (29) U/day, p $<0.001)$. Insulin remained unchanged in group B (oral 89\%; insulin 67\%, 15 (14) v 16 (15) U/day at visit 1$)$ and metformin was increased (56\% v 11\%). At visit 2, fasting $\beta$ glucose had decreased significantly by 3.8 (2.6) $\mathrm{mmol} / \mathrm{l}$ in group A and $3.6(2.8) \mathrm{mmol} / \mathrm{l}$ in group B $(\mathrm{p} \leqslant 0.01)$. Heart rate, blood pressure, and rate-pressure product remained unchanged.

The echocardiograms showed normal left ventricular global systolic function in all patients. Diastolic myocardial velocity at rest increased from visit 1 to visit 2 in group A (7.5 (1.7) $\mathrm{cm} / \mathrm{s}$ to $8.6(1.8) \mathrm{cm} / \mathrm{s}, \mathrm{p}<0.001$, fig l) but remained unchanged in group B. Systolic velocity remained unchanged in both groups. Changes in diastolic resting velocity correlated with changes in fasting $\beta$ glucose $(r=0.53$, $\mathrm{p}<0.002)$ and with changes of insulin dose $(r=0.47$, $\mathrm{p}<0.006)$. This pattern persisted when patients with and without evidence for coronary artery disease were looked at separately (fig 2).

The capillary blood volume index at maximum vasodilatation increased in both groups $(\mathrm{p}<0.02)$ (fig 1$)$. This change was significantly correlated with the decrease of fasting $\beta$ glucose $(r=0.52, \mathrm{p}<0.002)$ both in patients with and in patients without evidence for coronary artery disease (fig 2 ). In the controls, all parameters remained unchanged.

\section{DISCUSSION}

The noted effect of augmented diastolic function correlated with improved glycaemic control both in patients with and in patients without evidence of ischaemic heart disease. Although the exact pathophysiological mechanisms remain unknown, improved myocardial energy substrate utilisation is likely to be involved. Another potential mechanism is augmented microvascular perfusion. Endothelial dysfunction is aggravated by hyperglycaemia and may be partially reversed by insulin. Regulation of recruitable capillaries is influenced by hyperglycaemia and by insulin. ${ }^{4} \mathrm{~A}$ reasonable assumption is that effects on endothelial function are involved as shown by forearm blood flow with intensive insulin based treatment in patients with non-insulin dependent diabetes ${ }^{5}$ and by the present study for the first time in the myocardium as well.

This pilot investigation has the disadvantage related to observational studies of patients who were recruited from clinical practice with intensified antidiabetic treatment based on clinical needs. Such a study design may cause selection bias but has provided a possibility for initial testing of the hypothesis that insulin based metabolic control would exert beneficial effects.

The findings in this study are new and warrant confirmation in future randomised trials. From the present observations it is reasonable to assume that normoglycaemia and insulin improve myocardial diastolic function and perfusion in non-insulin dependent diabetes. Moreover, the findings support the hypothesis that cardiac abnormalities reported in diabetes mellitus contain a component of reversibility. Taken together this may have important future treatment implications.

\section{ACKNOWLEDGEMENTS}

This study was supported by grants from the Swedish Heart-Lung Foundation, King Gustaf V and Queen Victoria Foundation, and AFA Insurance. Drs Hansen and Dounis were supported by grants from the German Cardiac Society and the Hellenic airforce, respectively.

\section{Authors' affiliations}

H von Bibra, A Hansen, V Dounis, K Malmberg, L Rydén, Department of Cardiology, Karolinska Hospital, Stockholm, Sweden

T Bystedt, Department of Endocrinology, Karolinska Hospital, Stockholm, Sweden

Correspondence to: Professor Helene von Bibra, Department of Endocrinology, Academic Hospital München-Bogenhausen, Englschalkingerstrasse 77, 81925 Munich, Germany; von-Bibra@ extern.lrz-muenchen.de

Accepted 12 March 2004

\section{REFERENCES}

1 Hansen A, Johansson BL, Wahren J, et al. Beneficial effects of C-peptide on myocardial function in patients with type 1 diabetes. Diabetes 2002;51:3077-82.

2 Von Bibra H, Bone D, Niklasson U, et al. Myocardial contrast echocardiography yields best accurracy using quantitative analysis of digital data from pulse inversion technique: comparison with second harmonic imaging and harmonic power Doppler during simultaneous dipyridamole stress SPECT studies. Eur J Echocardiogr 2002;3:271-82.

3 Wei K, Jayaweera AR, Firoozan S, et al. Quantification of myocardial blood flow with ultrasound-induced destruction of microbubbles administered as a constant venous infusion. Circulation 1998;97:473-83.

4 Vincent MA, Dawson D, Clark A, et al. Skeletal muscle microvascular recruitment by physiological hyperinsulinemia precedes increases in total blood flow. Diabetes 2002;51:42-8.

5 Rask-Madsen C, Ihleman N, Krarup T, et al. Insulin therapy improves insulinstimulated endothelial function in patients with type 2 diabetes and ischemic heart disease. Diabetes 2001;50:2611-8. 